\title{
Tolerance is not a virtue
}

DOI:

10.1038/nrm2155

URLs
ATM
http://ca.expasy.org/uniprot/

Q13315

Artemis

http://ca.expasy.org/uniprot/

Q96SD1

Ataxia-telangiectasia

http://www.ncbi.nlm.nih.gov/

entrez/dispomim.

cgi?id=208900

RS-SCID

http://www.ncbi.nlm.nih.gov/

entrez/dispomim.

cgi? id $=602450$
Patients with underlying cellular defects in the response to DNA double-strand breaks (DSBs) often exhibit genomic instability, increased cancer predisposition and radiation sensitivity. Jeggo, Löbrich and colleagues have sought to dissect how DSB-induced checkpoint activation and repair in cycling cells influence these clinical characteristics.

Previously, it was shown that a subset of ionizing radiation (IR)-induced DSBs are repaired in an ataxia-telangiectasia mutated (ATM)- and artemis-dependent subpathway of non-homologous end-joining (NHEJ) in non-cycling cells. Artemis-deficient cells exhibit a DSB-repair defect, whereas cells derived from a patient with ataxiatelangiectasia (A-T; deficient for ATM activity) show both DSB-repair

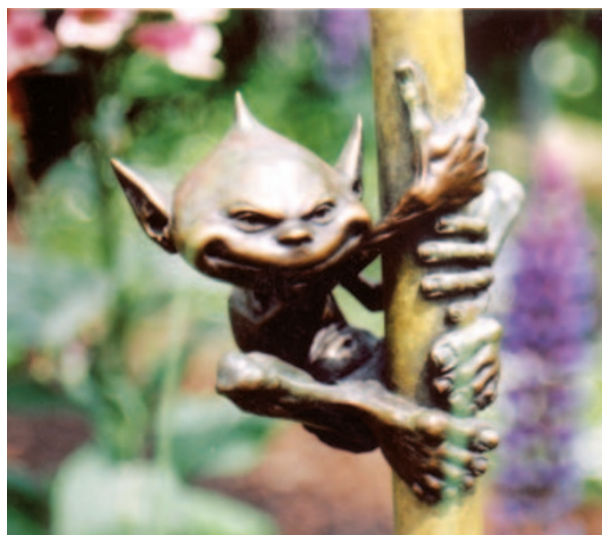

and cell-cycle checkpoint arrest defects.

Using primary fibroblasts derived from patients with A-T or RS-SCID (severe combined immunodeficiency with sensitivity to ionizing radiation; deficient for artemis activity) and $\mathrm{Atm}^{-/-}$or artemis ${ }^{-/-}$mouse embryonic fibroblasts, Jeggo, Löbrich and colleagues showed that a subset of DSBs require repair by the ATM-artemis arm of NHEJ in both G1- and G2-phase cells exposed to X-rays. However, RS-SCID cells showed a delay in G2-M checkpoint deactivation compared with normal cells, indicating that checkpoint activation is maintained to provide time for DSB repair. Approximately 15\% of IR-induced DSBs persisted considerably longer than the time it takes for the checkpoint to be deactivated in artemis-deficient cells, indicating that DNA damage is transmitted to mitosis and the following G1 phase. Mitotic, irradiated A-T cells had more chromosome breaks than equivalent RS-SCID cells, which in turn had more breaks than normal cells, demonstrating the influence of both the repair and checkpoint defects on genomic instability.

Interestingly, both normal and RS-SCID mitotic cells showed persistent DSBs after deactivation of the G2-M checkpoint, indicating that the maintenance of ATM-dependent
G2-M checkpoint arrest does not fully correlate with the completion of DSB repair. Next, the authors investigated the kinetics of mitotic chromosome breakage and showed that mitotic DSBs increased when cells entered mitosis after G2-M checkpoint deactivation compared with checkpoint escape, as is the case for checkpoint-defective A-T cells. Importantly, they showed that both RS-SCID and normal cells that have undergone G2-M checkpoint arrest have 10-20 DSBs in mitosis. This provides direct evidence for a threshold number of DSBs that are required to activate the ATM-dependent G2-M checkpoint. Furthermore, exposure of cells to radiation doses that result in less than 20 DSBs failed to activate the G2-M checkpoint.

These data indicate that the G2-M checkpoint is activated by a threshold level of DNA damage, potentially indicating the importance of the G1-S checkpoint in preventing the transmission of damaged DNA. Genomic instability is a common characteristic of cancer cells and might, in part, be propagated by such threshold tolerance to DNA damage.

Gemma Alderton, Associate Editor, Nature Reviews Cancer

ORIGINAL RESEARCH PAPER Deckbar, D. et al. Chromosome breakage after $\mathrm{G} 2$ checkpoint release.J. Cell Biol. 12 Mar 2007 (doi:10.1083/ jcb.200612047) 